\title{
Complejidad Social y Educación Superior. Análisis Crítico Basado en Agentes
}

\author{
Eduardo Ahumada-Tello \\ eahumada@uabc.edu.mx \\ https://orcid.org/0000-0003-1698-5126 \\ Universidad Autónoma de Baja California \\ Karen Ramos \\ karen.ramos38@uabc.edu.mx \\ https://orcid.org/0000-0002-5760-1419 \\ Universidad Autónoma de Baja California
}

Recepción: 30/09/2021

Aceptación: 20/10/2021

\section{Resumen}

El presente articulo tiene como finalidad llevar a cabo el desarrollo de una propuesta de análisis crítico de la evolución social y la educación desde una perspectiva de complejidad. Para esto, se inicia con la conceptualización del conocimiento como valor intrínseco de los individuos y las organizaciones, por lo que su desarrollo va gestando la construcción y comprensión de lo que se conoce como "Sociedad del Conocimiento". Se hace una aproximación teórica sobre los procesos evolutivos que afectan a la sociedad y como estos dan paso al uso de la tecnología y la innovación en este nuevo orden social que finalmente impacta en la educación. Así mismo, se lleva a cabo el desarrollo de un caso de estudio utilizando agentes para evaluar el proceso de resolución de problemas en la Sociedad del Conocimiento 5.0 en una institución de educación superior, esto ejemplifica la necesidad de abordar el estudio con un enfoque de complejidad, eliminación de entropía y sostenibilidad. Cabe señala que en este caso, los agentes (profesor y alumno) utilizan principios BDI y se apegan a la biblioteca de Sakellariou (2008). Finalmente se observa que, bajo los parámetros ingresados de manera empírica, aproximadamente un 15 por ciento llega a la generación de conocimiento, se debe considerar que este resultado puede variar si las instituciones definen políticas y acciones a tomar con el fin de incrementar la motivación y disposición de los estudiantes hacia el proceso de creación de conocimiento.

\section{Abstract}

The purpose of this article is to develop a proposal for a critical analysis of social evolution and education from a complexity perspective. For this, it begins with the conceptualization of knowledge as an intrinsic value of individuals and organizations, so its 
development is conceiving the construction and understanding of what is known as the "Knowledge Society". A theoretical approach is made on the evolutionary processes that affect society and how these give practices to the use of technology and innovation in this new social order that ultimately impacts education. Likewise, the development of a case study is carried out using agents to evaluate the problem-solving process in the Knowledge Society 5.0 in a higher education institution, this exemplifies the need to consider the study with a complexity approach, entropy elimination and sustainability agenda. It should be noted that in this case, the agents (teacher and student) use BDI principles and adhere to the Sakellariou library (2008). Finally, it is observed that, under the parameters entered empirically, approximately 15 percent reaches the generation of knowledge, it should be considered that this result may vary if the institutions define policies and actions to take in order to increase motivation and disposition of students towards the process of knowledge creation

Palabras Clave: Sociedad del Conocimiento 5.0; Complejidad; Agentes; BDI

Keywords: Knowledge Society 5.0; Complexity; Agents; BDI

\section{Introducción}

El desarrollo de las ciencias computacionales ha logrado mejorar las herramientas que se pueden utilizar para abordar para tratar, primero de defirnir, conceptualizar, diseñar una aproximación metodológica, construir y comprender un determinado problema social. En el crecimiento y evolución de los grupos sociales, ha aparecido en tiempos recientes, un nuevo valor que cada individuo, organismo y gobierno buscan: el conocimiento como eje fundamental que provea elementos de competitividad y que mejoren el desempeño de los organimos y las empresas de un espacio determinado o de una economía en particular (Minati, 2016).

En este documento se abordan los temas de evolución del grupo social, las implicaciones que esto tiens para la sostenibilidad de las estrategias que permitan ampliar el periodo de influencia que este grupo pueda desarrollar. Tambien se lleva a cabo un análisis de los constructos de Sociedad del Conocimiento 5.0, Educación 5.0 y su importancia para aprovechar las condiciones actuales que se presentan en un ambiente global (Zhang et al., 2021).

En la sección del desarrollo de la metodología se explica el uso de la herramienta de casos de uso y de etnografía, así como la determinación que el estudio por realizar será en la Universidad Autónoma de Baja California bajo parámetros empíricos elegidos aleatoria- mente. Estos datos dan pie al desarrollo de un modelo inicial en la herramienta NetLogo que permite predecir el resultado de la aplicación de condiciones iniciales empíricas, acciones condicionadas y resultados obtenidos, esto obedeciendo al modelo de Agentes BDI que son explicados durante las referencias teóricas en el documento.

El documento presenta entonces una serie de pasos que pueden resumirse de la siguiente manera:

I. Definición de los conceptos teóricos que sostienen la relación entre evolución social, sociedad del conocimiento, sociedad del conocimiento 5.0, educación 5.0.

II. Metodologica de estudio de casos aplicado al desarrollo de datos empíricos.

III. Modelo de agentes BDI basado en la complejidad inherente al proceso educativo

IV. Conclusiones y propuestas futuras

\section{Aproximaciones teóricas}

\section{a. Evolución social}

La evolución natural de un grupo social involucra un crecimiento similar al de un producto. En sus diferentes fases se construyen las bases y fundamentos morales, sociales, económicos, políticos y comerciales para la interacción entre sus miembros y sus pares. Esta primera fase, se define en que es lo que sucederá en el futuro próximo, que es en donde se presentará una interacción de alto nivel con otros grupos sociales circundantes, siendo por consecuencia la segunda parte, en la que por correspondencia crece su nivel de influencia con los demás. En la parte tres, esa influencia tiende a mantenerse por un periodo variable y que depende de la manera en que se logra perpetuar todo el fundamento ideológico-político entre sus agentes interesados (Omodeo, 2019). Finalmente, ocurre un fenómeno natural entrópico que ocasiona que el enramado social pueda debilitarse e iniciar un proceso de degeneración y disminución de su cohesión social así como su relación con pares (Diamond, 1997). Este proceso se ilustra en la Figura 1

Cada fase indicada en la Figura 1, indica las características de las etapas evolutivas mencionadas. En ello se observa que a partir de la conformación de un grupo social, este pasa por varias transiciones que le llevan a desarrollar su propia estrategia para proyectar una permanencia sostenible y sustentable en el ámbito regional y global. Bajo estos conceptos, es factible predecir el desarrollo y la etapa de influencia que un grupo social tiene internamente y además que implementa hacia sus vecinos 


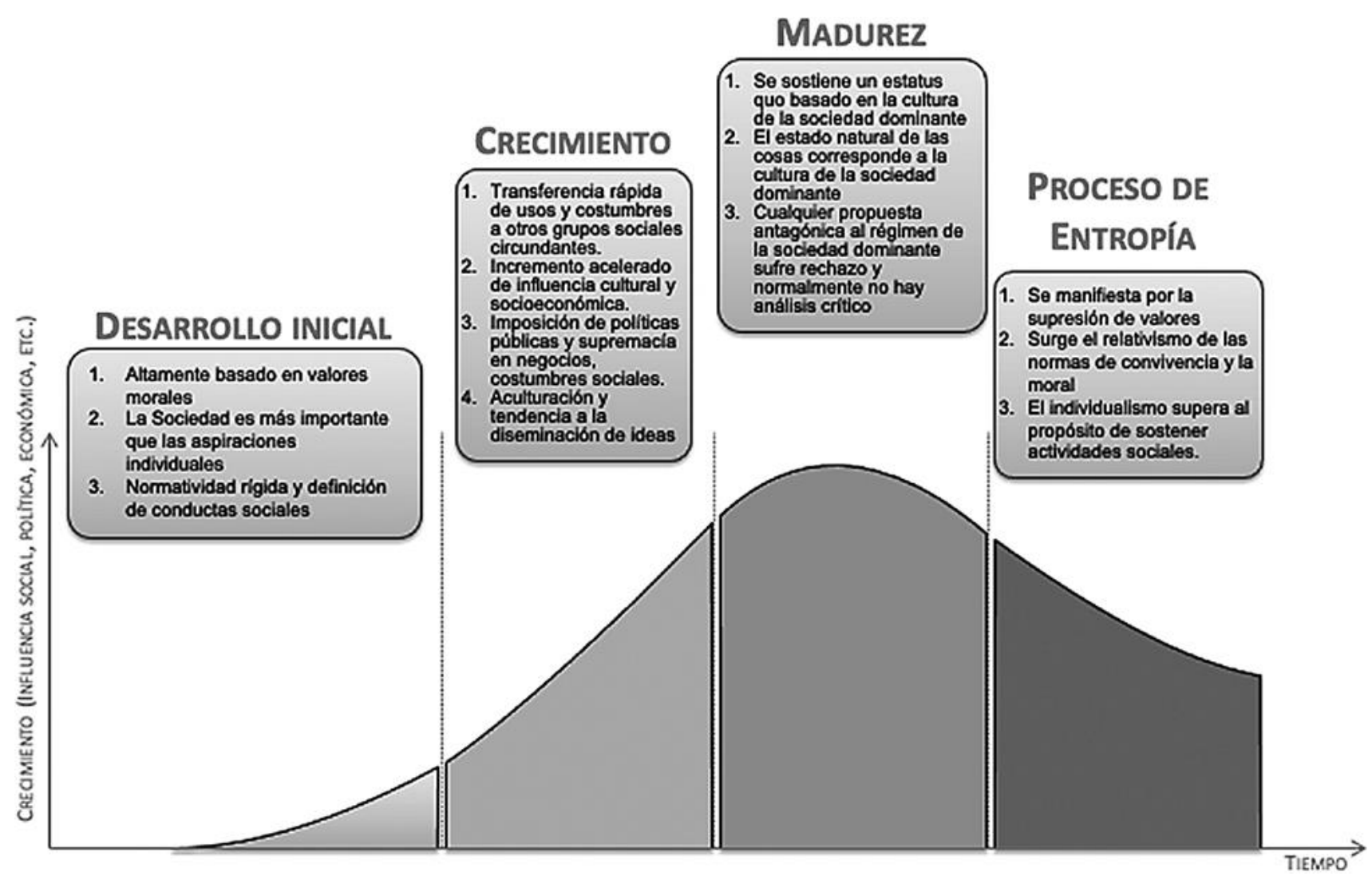

Fuente: Elaboaración propia

locales, regionales y globales. Ante esta disyuntiva, sería normal observar una disminución de los efectos e influencia en la evolución social a través tiempo. Esto provocaría que una sociedad determinada pueda ser sustituidos por otra que se encuentren en una fase con mayor cohesión social, como se observa, este proceso emula de manera casi idéntica al ciclo de vida del producto, lo que permite detectar un posible modelo inicial probado en otro dominio del conocimiento que se puede transferir y aplicar para el estudio de la evolución de las sociedades y encontrar elementos claves que facilitan el análisis crítico orientado a la prospectiva social (Skorodumova et al., 2016).

\section{b. Sociedad del conocimiento}

Uno de los fenómenos mas notables en los que han incurrido los grupos sociales modernos para mantener durante el mayor tiempo posible su permanencia como líderes socioeconómicos y en esta tendencia, la posibilidad de generar una influencia sostenible sobre sus miembros y sus pares, es la revolución tecnológica de la sociedad. Desde los primeros pasos cuando los procesos de producción estaban basados en los recursos naturales y en la fuerza física de los habitantes, hasta el momento actual en el que se mantiene una permanente actitud innovadora y emprendedora para diversificar los medios de producción que actualmente se basan en el conocimiento (Castells, 2002).

Esta tendencia enfocada en construir puentes entre los procesos productivos que se fundamenten en la creación de valor a partir del conocimiento no es nueva. Anteriormente se ha logrado determinar que este viaje social tiene sus orígenes en culturas antiguas y que se mantiene como una conducta repetible y permanente en el surgimiento constante de nuevas alternativas culturales, políticas, sociale sy económicas para sostener la existencia de la especie humana (Diamond, 1997).

Es bajo esta perspectiva, que tradicionalmente podemos encontrar un avance similar y relacionado entre si, con la evolución de los grupos sociales y su manera de crear opciones cada vez mas tecnificadas de los medios de producción a los que se tenga acceso. Es por ello que en la Figura 2, se identifica rápidamente este fenómeno aplicado a la era moderna. Donde se puede detectar sin problema alguno, el momento histórico en el cual se modificaron los medios de producción central para el desarrollo de la sociedad moderna. 
Figura 2. Representación evolutiva de la sociedad del conocimiento

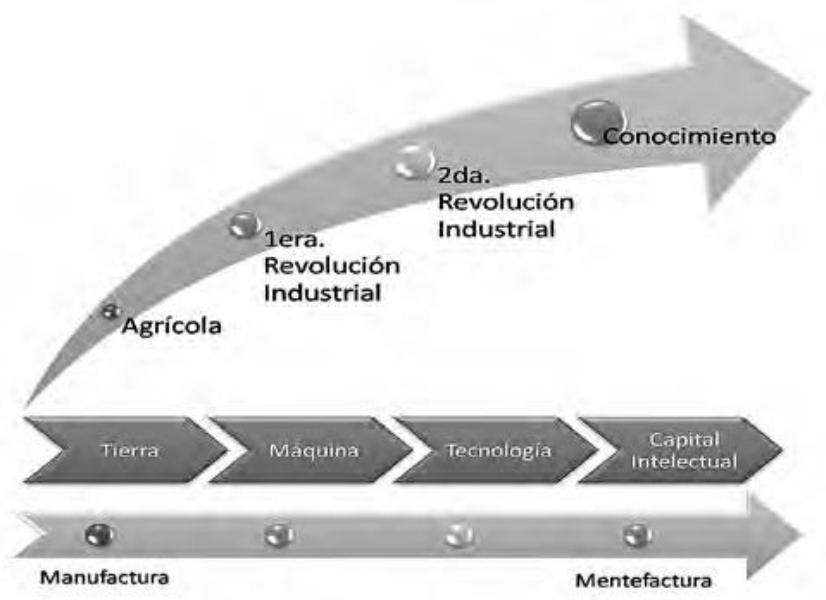

Fuente: Elaboración propia con base en (Villarreal, 2006)

Pero no hay que olvidar que este fenómeno es también parte de la historia de la humanidad, el desarrollo de avances tecnológicos y la dependencia en el conocimiento no es exclusivo de nuestra era. La construcción de chinampas como medio de producción agropecuaria en el lago de Tenochtitlan es un ejemplo fehaciente de la importancia del desarrollo tecnológico aplicado a recursos limitados y aprovechando al máximo las condiciones que se tienen. Existen muchos otros ejemplos: la construcción de las pirámides de Egipto, los jardines colgantes de Babilonia, los acueductos romanos, el sistema de conteo administrativo implementado por los Incas para mantener supervisada su producción agropecuaria, el desarrollo matemático y astronómico de los Mayas y de los árabes, el descubrimiento de la pólvora en China, el autosuministro de agua por parte de los aborígenes australianos. Y un sinfín de ejemplos que manifiestan que el ser humano como individuo y como miembro de un grupo social, siempre se encuentra a la búsqueda de aprovechar los recursos que tiene a su alcance y para lograrlo tiene la tendencia permanente a utilizar medios innovadores y tecnológicos que le permitan hacer esto de manera eficiente (Diamond, 1997).

\section{c. Sociedad del conocimiento $\mathbf{5 . 0}$}

Al enfocar este trabajo en el desarrollo moderno, es importante hacer referencia al surgimiento del concepto de Sociedad de Conocimiento 5.0, este surge principalmente en Japón y hace referencia a una nueva dinámica y enfoque que se sustenta en la tecnología y su relación directa con el estado de bienestar en una sociedad específica.
La tecnología moderna, basada en elementos electrónicos está cada vez más presente en todos los sectores de la sociedad, por lo que hoy en día varios tecnologías y herramientas digitales en la enseñanza, en el comercio, los servicios y en los más variados sectores de la sociedad se han convertido en una realidad permanente y trivial. La tecnología cambia a pasos agigantados y de la misma manera, se está generando una cantidad impresionante de información que mejora la predicción de comportamientos individuales [compra, venta, compromiso personal y familiar, etc.], como sociales [tendencias políticas, movimientos sociales, promoción de leyes y fundamentación del cambio en el entorno geopolítico global, etc.] (Skorodumova et al., 2016).

Un aspecto importante a considerar es que la industria/sociedad 4.0 y la 5.0 están relacionados con las revoluciones industriales y tecnológicas, por lo que la sociedad 4.0 es un producto de la cuarta revolución industrial, mientras que la sociedad 5.0 se ve como una evolución en proceso. Esto influye en todos los términos y conductas comunes, como en la educación, la cultura, los negocios, los valores fundamentales de las relaciones humanas y las leyes que protegen las actividades comerciales, las políticas públicas y las consideraciones que surgen para el ajuste de los valores morales, entre otros. Para diferenciar lo mas asertivo posible las implicaciones de estos dos conceptos, es recomendable que puntualicemos cuáles fueron los advenimientos de cada uno de estos fenómenos (John et al., 2020).

La cuarta revolución industrial, también llamada Industria 4.0 o Sociedad 4.0, está marcada por avances tecnológicos como:

- Robótica

- Comunicación digital

- Almacenamiento en la nube

- Internet de las Cosas

Cuando pensamos en los frutos cosechados por estos avances tecnológicos, aquí es donde comenzamos a entender en qué consiste el concepto de Sociedad 5.0. Por lo tanto, la revolución tecnológica y digital vivida en Sociedad 5.0 está guiada por avances tecnológicos como:

- Big data

- Ciudades inteligentes

- Educación 5.0

- Casas inteligentes

- Medicina robotizada

-

Retomando el proemio de esta sección, en donde se establece que el origen conceptual de la So- 
ciedad del Conocimiento 5.0 y sus subsecuentes lineamientos, es en Japón, en donde a partir de 2016 se establece un plan de inversión gubernamental nombrado "Quinto plan básico de ciencia y tecnología", en donde el gobierno enumera una serie de políticas e innovaciones que le país debe elaborar de manera anual. Es en este plan en donde surge el concepto de Sociedad 5.0. Con esto, se pretende que, mediante el uso de la tecnología, se cubran necesidades esenciales como la salud, el bienestar social, la asistencia médica de mergencia, la paz, el trabajo, entro otros, haciéndolo extensivo a toda la población mundial, no solo a los de mejores ingresos, tanto individuales como nacionales. Esta iniciativa ha sido analizada por otros países y poco a poco se va formando un nuevo hito evolutivo social.

Algunos de las problemáticas que fueron mencioandas por el gobierno japonés se pueden enumerar en la tabla 1, donde se pone de manifiesto la necesidad de mejorar el entorno social que conlleve a solucionar problemas que ocasionan polarización entre los pueblos y las personas.

Tabla 1. Resolución de Problemas en la Sociedad del Conocimiento 5.0

\begin{tabular}{|l|l|}
\hline \multicolumn{1}{|c|}{ Problemas } & \multicolumn{1}{c|}{ Soluciones } \\
\hline Mayor demanda de energía & $\begin{array}{l}\text { Reducción de gases de efecto } \\
\text { invernadero }\end{array}$ \\
\hline Mayor demanda de alimentos. & $\begin{array}{l}\text { Reducción de residuos y aumento } \\
\text { de la producción. }\end{array}$ \\
\hline Competicion internacional & $\begin{array}{l}\text { Industrialización saludable y } \\
\text { renovable }\end{array}$ \\
\hline $\begin{array}{l}\text { Concentración de ingresos y } \\
\text { riqueza }\end{array}$ & $\begin{array}{l}\text { Redistribución de ingresos y alivio } \\
\text { de la pobreza }\end{array}$ \\
\hline
\end{tabular}

Fuente: (Suki Desu, 2018)

Por tanto, según los creadores de este concepto en el gobierno japonés, la tecnología debe utilizarse en el bienestar social, la distribución equitativa y juste de la renta y la riqueza, la reducción del impacto y daño ambiental, buscando, de esta manera, promover la igualdad social, el bienestar ambiental y la longevidad de la población y de la sociedad.

\section{d. Educación 5.0}

En el ámbito educativo y adicionando el bienestar social que promueve esta nueva revolución tecnológica moderna, se prevee que la educación sea impactada y transformada de una manera positiva y con la expectativa de que también sea sostenible. Esto se debe a que, en los nuevos modelos educativos a lo que se le llama Educación 5.0, toma en cuenta el bienestar psicoemocional de los estudiantes y de la comunidad académica, docente y administrativa en general (Macintyre et al., 2020).

Es de esperarse que en este nuevo concepto se incluyan conceptos ambientales, enfoque en valores individuales y sociales, incremento de la participación cívica, inclusión, diversidad, empatía con las minorías y aceptación de diferencias, amplitud del estudio analítico crítico fundamentado en procesos científicos entre otros y utilizando recursos tecnoloógicos que ya se encuentran en operación actualmente (Bashiri et al., 2017).

\section{Metodología de estudio de caso}

La elección de esta metodología de investigación cualitativa, se debe a su función de ayudar a comprender con profundidad las dinámicas presentes dentro de escenarios individuales y a descubrir nuevas relaciones y conceptos en ellos, su aplicación se enfoca en la comprensión más que en la comprobación o validación de presuposiciones previas (Bernal, 2012).

La etnografía es la rama de la antropología dedicada a la observación y descripción de los distintos aspectos de una cultura o pueblo determinado, como el idioma, la población, las costumbres y los medios de vida (Harrison et al., 2020). Todo ello con la finalidad de lograr comprender el fenómeno que se estudia, aplica a la actividad de generar un constructo basado en esta metodología.

Los estudios de caso son una metodología cualitativa similar a la etnografía. Los investigadores de estudios de caso se enfocan en un programa, evento o actividad que envuelve individuos más que a grupos de individuos. Existe en ello también un interés en describir el proceso más que en identificar patrones de conducta exhibidos por el grupo (Creswell, 2002). Los objetivos que se persiguen en la investigación cualitativa con la aplicación de casos de estudio son los siguientes:

- Analizar el fenómeno existente sobre la concepción de agentes y sus caracteríticas de Creencias (B), Deseos (D) e Intensiones (I), aplicados a un problema sustentado en el desarrollo de la sociedad del conocimiento 5.0 y su proceos educativo corresponiendte.

- Identificar los elementos y factores que determinan el desarrollo de la sociedad del conocimiento 5.0 específicamente en el ámbito educativo.

Bajo estas finalidades, se propone dar inicio a la construcción de una linea de investigación que aborde los temas relacionados con la generación de conocimiento, desarrollo de sociedades que respondan a la evolución social natural de cada grupo y que, mediente 
el uso de tecnología, innovación e investigación mejoren las condiciones para manejar un futuro sostenible desde una apreciación basada en la complejidad de las interacciones que se presenten.

\section{Sistemas Complejos basados en Agentes}

\section{a. Caso de estudio}

Los sistemas complejos exhiben propiedades que surgen de la interacción de sus partes y que no se pueden predecir a partir de las propiedades independientes que estas posean (Kapsali et al., 2021). Estos sistemas constan de muchos componentes o partes diversos y autónomos pero interrelacionados e interdependientes vinculados a través de muchas interconexiones, por lo tanto, no pueden describirse con una sola regla y sus características no se pueden reducir a un nivel de descripción por el elevado número de ellas. Es necesario hacer un análisis en profundidad de sus propiedades y características específicas para mejorar el entendimiento de su comportamiento (Gilbert \& Troitzsh, 2008; Wooldridge $\&$ Jennings, 1995).

El propósito de modelar un programa educativo es que a través de este proceso el investigador sea capaz de identificar los elementos que se pueden intervenir para incrementar la calidad de los resultados esperados del mismo. En este contexto, en esta sección del documento se tiene como objetivo representar el proceso educativo y de investigación en Instituciones de Educación superior a través del análisis de los elementos en una investigación aplicada en la Universidad Autónoma de Baja California en Tijuana México. Los agentes y variables propuestos e involucrados en esta investigación, tienen los siguientes agentes y variables en este modelo propuesto (Ahumada-Tello et al., 2011, 2018; Ahumada-Tello \& Castanon-Puga, 2016; Diamond, 1997; Suarez \& Castañón-Puga, 2013):

Tabla 2. Resolución de Problemas en la Sociedad del Conocimiento 5.0

\begin{tabular}{|l|l|}
\hline \multicolumn{1}{|c|}{ Agentes } & \multicolumn{1}{c|}{ Variable de estudio } \\
\hline Estudiantes, Profesores y Programas & Proceso enseñanza-aprendizaje \\
\hline $\begin{array}{l}\text { Investigación, desarrollo tecnológico, } \\
\text { innovación }\end{array}$ & Investigación y desarrollo \\
\hline Gestión de recursos, tiempos y personal & Gestión \\
\hline Ambiente y agentes externos & Interesados \\
\hline
\end{tabular}

Fuente: Elaboración propia
En la Tabla 2 se clasifican a los agentes en cuatro categorías, pero con un total de diez diferentes entidades que se dividen en: 1 . Proceso enseñanza-aprendizaje; 2. Investigación y desarrollo; 3. Gestión; y, 4. Ambiente. Se propone entonces analizar a los involucrados en el proceso desde los participantes directos en la transferencia del conocimiento, la investigación, la gestión y los interesados en el resultado final.

\section{b. Agentes y complejidad}

El término agente es muy utilizado, por varios investigadores, estudiantes y profesores de diversas áreas del conocimiento. Por lo general, se usa este término para referirse a un sistema informático que, además de tener propiedades de conducta y comportamiento, se conceptualiza o implementa utilizando conceptos que generalmente se aplican a los humanos (Wooldridge \& Jennings, 1995).

Por ejemplo, en inteligencia artificial es habitual caracterizar a un agente utilizando nociones sociales, como conocimiento, creencia, intención y obligación. Algunos investigadores incluso han considerado incluir agentes emocionales. Otra forma de dar a los agentes atributos similares a los humanos es representarlos visualmente (Gilbert \& Troitzsh, 2008).

Este es un enfoque basado en el estudio de las actitudes mentales y aborda los problemas que surgen al intentar utilizar la planificación tradicional en situaciones que requieren reactividad en tiempo real. La "B" significa Creencias que representan el estado informativo de un agente, es decir, lo que sabe sobre sí mismo y el mundo. La "D" significa Deseos o metas son su estado motivacional, es decir, lo que el agente está tratando de lograr. Un agente típico tiene el llamado conocimiento procedimental constituido por un conjunto de planes que definen secuencias de acciones y pasos a realizar para lograr un determinado objetivo o reaccionar ante una situación específica. Finalmente, la "I" significa intenciones que representan el estado deliberativo del agente, es decir, qué planes ha elegido el agente para su eventual ejecución (De Camargo et al., 2020; Rovbo \& Ovsyannikova, 2020).

\section{c. Modelo computacional}

En este documento se incluye una propusta inicial para la simulación basada en agentes de un modelo educativo utilizando las características de agente BDI (Ahumada-Tello \& Castanon-Puga, 2016).

Creencias: El modelo asume que la facultad tiene un número de profesores de tiempo completo acorde con el número de estudiantes, además 
cuenta con la infraestructura suficiente. Al final se asume que la escuela cuenta con aulas, laboratorios de computación, movilidad académica, prácticas, servicio social, pasantías y actividades culturales y deportivas. El profesor horario de trabajo. Para el docente: horas docencia, investigación, gestión y estrategias. Este último considera que cuentan con formación profesional y pedagogía para transmitir la competencia como facilitador del aprendizaje de los estudiantes. Para los estudiantes: la motivación (student-mot) y la disposición al estudio (student-disp) dependen tanto de su interacción con los profesores como entre ellos.

Deseos: Los objetivos de la simulación son aumentar la capacidad de mejorar el aprendizaje y la generación de conocimientos por parte del alumno. Asumimos que cuando el alumno logra las habilidades de aprendizaje también está generando conocimiento, esta es una medida por encima del $90 \%$ de las competencias.

Intenciones: Las acciones a realizar por los agentes son: Estudiante: student-learning (estudiante-aprendizaje), student-knowledge (estudiante-conocimiento); Profesor: r-professor (investigación), $\mathbf{m}$-professor (gestión), t-professor (enseñar). Las intenciones anteriores cubren los requisitos para que el docente transmita las habilidades necesarias que permitirán la generación de conocimiento

En la Tabla 3, se desriben los elementos del proceso educativo que se consideran para realizar el proceso de programación del modelo basado en agentes BDI utilizando la herramienta NetLogo y estableciendo valores iniciales para la simulación.

Tabla 3. Datos iniciales, acciones y salidas del modelo BDI educativo

\begin{tabular}{|l|l|l|}
\hline \multicolumn{1}{|c|}{ Datos iniciales } & \multicolumn{1}{|c|}{ Acciones } & \multicolumn{1}{c|}{ Salidas } \\
\hline Student Qty: 150 & t-professor: 20 & student-learning \\
\hline Professor Qty: 15 & r-professor: 8 & knowledge-generation \\
\hline Time: 80 (days) & m-professor: 12 & \\
\hline & $\begin{array}{l}\text { student_disp: } 60 \\
\text { student_mot: } 60\end{array}$ & \\
\hline
\end{tabular}

Fuente: Elaboración propia

En la Figura 3, se desriben los elementos del proceso educativo que se consideran para realizar el proceso de programación del modelo basado en agentes BDI utilizando la herramienta NetLogo. Así mismo, se muestran los elementos que afectan el proceso y los resultados esperados al combinarse las acciones tomadas por profesores y estudiantes.

Siempre $\mathrm{m}$-profesor $+\mathrm{r}$-profesor $+\mathrm{t}$-profesor debe ser igual a 40 (Cantidad de horas que trabaja
Figura 3. Acciones a tomar en el proceso de simulación

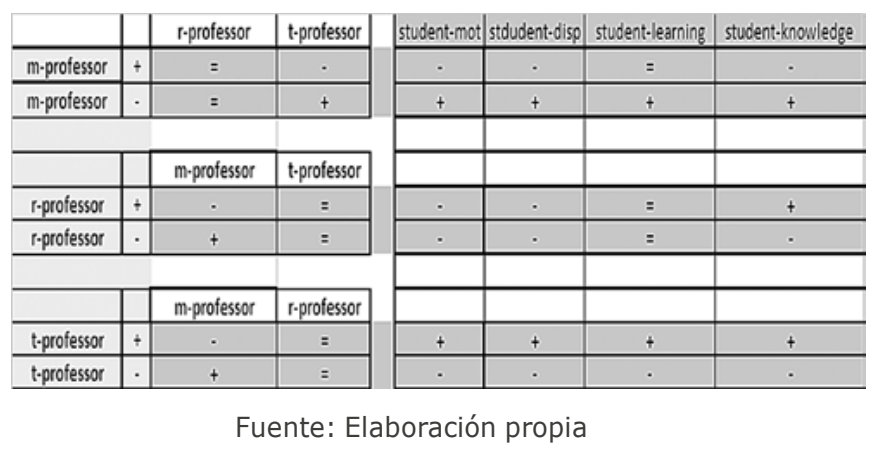

un profesor) o igual a 90, incrementamos el conocimiento del alumno. También evaluamos el aprendizaje del estudiante cada 80 días (cada semestre)

El modelo de aprendizaje estudiantil se evalúa en un evento cada 80 horas (usualment al final del semestre para validar el estado de las habilidades logradas), y si el aprendizaje es mayor o igual a 90 , se genera conocimiento. Con la generación de conocimiento se restablecen objetivo como el incremento de la cultura del aprendizaje, la motivación y la disposición a sus valores originales, y se suman a un nuevo semestre en donde los estudiantes cursan nuevas materias. La figura 4 muestra la simulación en NetLogo e incluye la biblioteca "Agentes con creencias e intenciones" desarrollada en NetLogo (Sakellariou, 2008) donde se presentan los resultados de aprendizaje adquiridos durante un semestre y la generación de conocimiento durante ese semestre (es decir, cuántos estudiantes lograron una calificación mayor o igual a 90)

Figura 4. Simulación en NetLogo
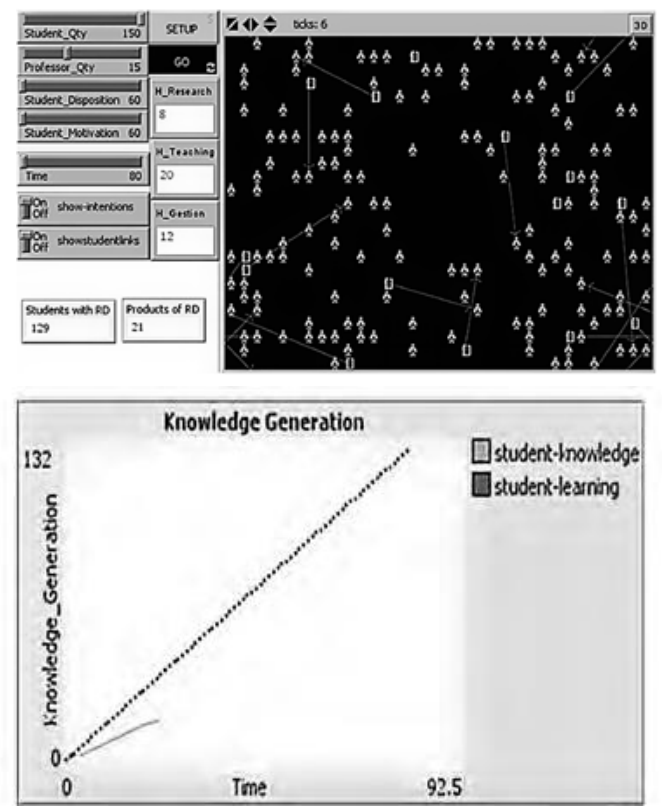

Fuente: Elaboración propia 


\section{Conclusiones}

En este artículo se ilustran los elementos evolutivos de la sociedad del conocimiento y se establece que para que un grupo social sea sostenible, es necesario que considere que todo grupo, ente, persona, producto y cosa "existente" entra en un proceso irreversible de entropía, lo que requiere que se consideren aspectos fundamentales para prospectar los efectos que se tienen en la generación de estrategias sostenibles que permitan lograr un desarrollo permanente y evolutivo de la sociedad.

De la misma forma, se aborda el tema de la educación desde un enfoque basado en agentes, en este caso estos agentes (profesor y alumno) tienen los principios BDI y utilizan la biblioteca de Sakellariou (2008) Se observa que bajo los parámetros ingresados de manera empírica, aproximadamente un 15 por ciento llega a la generación de conocimiento (alcanzando las competencias del modelo educativo), y este resultado puede variar si las instituciones definen políticas y acciones a tomar con el fin de incrementar la motivación y disposición de los estudiantes hacia el proceso. de la creación de conocimiento.

En trabajos futuros se propone la implementación de técnicas de lógica difusa para analizar y evaluar el proceso de aprendizaje alumno-profesor para saber si hay más acciones a tomar para incrementar la generación de conocimiento. También porque necesitará saber qué sucedió si un estudiante alcanza el 89,99 de aprendizaje y solo 90 o más alcanzan esa generación de conocimiento (Ervural et al., 2016). También es necesario implementar los otros factores que describimos anteriormente y pueden ayudar al modelo educativo en su objetivo de incrementar la adquisición de conocimientos (Hennessey \& Mueller, 2020; Idemudia et al., 2019).

Este caso de estudio se estable como uno de los importantes para desarrollar estrategias sostenibles que permitan el aletargamiento del proceso de entropía y lograr percibir que ocurre en el fenómeno del ciclo de vida social que mejore las condiciones para un crecimiento o mejor dicho, una permanencia en el entorno de mayor longevidad, al punto tal que al determinar la imposibilidad de detener el ocaso del grupo social, se esté perfilando la consolidación del siguiente.

\section{Referencias}

Ahumada-Tello, E., \& Castanon-Puga, M. (2016). Modelling complex systems with distributed agency and fuzzy inference systems. Knowledge-based curricula in higher education. Procedia Computer Science, 80. https://doi.org/10.1016/j. procs.2016.05.429

Ahumada-Tello, E., Castañón-Puga, M., Castro, J.-R., Suarez,
E. D., Márquez, B.-Y., Gaxiola-Pacheco, C., \& Flores, D.-L. (2011). On the multi-agent modelling of complex knowledge society for business and management system using distributed agencies. In Communications in Computer and Information Science: Vol. 188 CCIS (Issue PART 1). https://doi. org/10.1007/978-3-642-22389-1_49

Ahumada-Tello, E., Castanon-Puga, M., Evans, R. D., \& Gaxiola-Pacheco, C. (2018). Contributions of Knowledge Management to Firm Competitiveness from a Complexity Approach. 2018 IEEE Technology and Engineering Management Conference, TEMSCON 2018. https://doi.org/10.1109/TEMSCON.2018.8488416

Bashiri, H., Nazemi, A., \& Mobinidehkordi, A. (2017). Futures engineering in complex systems. Foresight, 19(3), 306-322. https://doi.org/10.1108/FS-09-2016-0042

Bernal, C. (2012). Metodología de la Investigación. Prentice-Hall.

Castells, M. (2002). La era de la información. Vol. I. La sociedad red. Plaza y Janés, S.A.

De Camargo, P. C., Mattos, S., \& Goldenberg, C. (2020). Complexity and collective intelligence on demand for a sustainable future. Proceedings - 14th IEEE International Conference on Semantic Computing, ICSC 2020, 347-349. https://doi. org/10.1109/ICSC.2020.00069

Diamond, J. (1997). Guns, germs and steel: The fates of human societies. W.W. Norton \& Company.

Ervural, B. C.., Ervural, B., \& Kahraman, C. (2016). Fuzzy sets in the evaluation of socio-ecological systems: An interval-valued intuitionistic fuzzy multi-criteria approach. Studies in Fuzziness and Soft Computing, 341, 309-326. https://doi. org/10.1007/978-3-319-31093-0_14

Gilbert, N., \& Troitzsh, K. G. (2008). Simulation for the Social Scientist. Open University Press.

Harrison, R. L., Reilly, T. M., \& Creswell, J. W. (2020). Methodological Rigor in Mixed Methods: An Application in Management Studies. Journal of Mixed Methods Research, 14(4), 473-495. https://doi.org/10.1177/1558689819900585

Hennessey, E., \& Mueller, J. (2020). Teaching and learning design thinking (DT): How do educators see DT fitting into the classroom? Canadian Journal of Education, 43(2), 498-521. https://www.scopus.com/inward/record. uri?eid=2-s2.0-85091719090\&partnerID $=40 \& \mathrm{md} 5=\mathrm{d}$ 7d215839ce6b5b9d78f2d42228e8671

Idemudia, E. C., Adeola, O., \& Achebo, N. (2019). The online educational model and drivers for online learning. International Journal of Business Information Systems, 32(2), 219-237. https://doi.org/10.1504/IJBIS.2019.103078

John, K. K., Adarsh, S. N., \& Pattali, V. (2020). Workers to super workers: A brief discussion on important technologies for industry 5.0 manufacturing systems. AIP Conference Proceedings, 2311. https://doi.org/10.1063/5.0034521

Kapsali, M., Bayer, S., Brailsford, S., \& Bolt, T. (2021). The agency role of simulation models in model-building groups. Journal of the Operational Research Society. https://doi.org/10.1080/0 1605682.2021.1929527

Macintyre, P. D., Ross, J., \& Clément, R. (2020). Emotions are 
motivating. In The Palgrave Handbook of Motivation for Language Learning. https://doi.org/10.1007/978-3-030-283803_9

Minati, G. (2016). Knowledge to manage the knowledge society: The concept of theoretical incompleteness. Systems, 4(3). https://doi.org/10.3390/systems4030026

Omodeo, P. D. (2019). Political epistemology: The problem of ideology in science studies. In Political Epistemology: The Problem of Ideology in Science Studies. https://doi.org/10.1007/9783-030-23120-0

Rovbo, M. A., \& Ovsyannikova, E. E. (2020). Methods of Local Behavior Planning for Agents with BDI Architecture. Scientific and Technical Information Processing, 47(6), 348-357. https://doi.org/10.3103/S0147688220060052

Sakellariou, I. (2008). Agents with beliefs and intentions in NetLogo. In A library of NetLogo.
Skorodumova, O. B., Matronina, L. F., \& Koval, T. I. (2016). Management in the knowledge society: Tendencies and Prospects. Indian Journal of Science and Technology, 9(12). https://doi. org/10.17485/ijst/2016/v9i12/89536

Suarez, E. D., \& Castañón-Puga, M. (2013). Distributed Agency. International Journal of Agent Technologies and Systems. https://doi.org/10.4018/jats.2013010103

Villarreal, R. (2006). Competitividad en la Era del Conocimiento. Centro de Capital Intelectual y Competitividad.

Wooldridge, M., \& Jennings, N. (1995). Intelligent Agents: Theory and Practice. Knowledge Engineering Review.

Zhang, W., Jiang, Y., \& Zhang, W. (2021). Capabilities for Collaborative Innovation of Technological Alliance: A Knowledge-Based View. IEEE Transactions on Engineering Management, 68(6), 1734-1744. https://doi.org/10.1109/TEM.2019.2936678 